\title{
Small Bowel Hematoma in the Setting of Concurrent Warfarin and Cannabis Use
}

\author{
Stephanie S Maldonado ${ }^{1 *}$ and Simon $\mathrm{K} \mathrm{Lo}^{2}$ \\ ${ }^{1}$ The Warren Alpert Medical School of Brown University, Providence, RI \\ ${ }^{2}$ Division of Digestive and Liver Diseases, Cedars-Sinai Medical Center, Los Angeles, CA
}

ISSN: 2637-7632

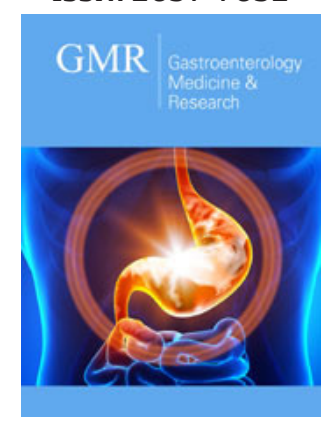

*Corresponding author: Stephanie S Maldonado, The Warren Alpert Medical School of Brown University, Providence, RI, USA

Submission: 侐 August 20, 2019

Published: 阱 September 05, 2019

Volume 3 - Issue 4

How to cite this article: Stephanie S Maldonado \& Simon K Lo. Small Bowel Hematoma in the Setting of Concurrent Warfarin and Cannabis Use. Gastro Med Res. 3(4). GMR.000568. 2019.

DOI: 10.31031/GMR.2019.03.000568

Copyright@ Stephanie S Maldonado, This article is distributed under the terms of the Creative Commons Attribution 4.0 International License, which permits unrestricted use and redistribution provided that the original author and source are credited.

\section{Introduction}

Anticoagulants are among the most commonly prescribed medications worldwide. With the legalization of marijuana across an increasing number of states, cannabis use has steadily increased nationwide in North America. There are limited studies on effects of natural cannabis on commonly prescribed anticoagulants such as vitamin $\mathrm{K}$ antagonists. The following case serves to educate on this potential interaction and encourage future studies given the prevalence of warfarin and marijuana and possible concomitant use in the United States. We present a case of a 53-year-old male on a stable dose of warfarin who presented to the emergency department with one week of progressively worsening lower abdominal and back pain, constipation and hematuria. He had a past medical history of stroke and interatrial septal aneurysm with patent foramen ovale. He began experiencing these acute gastrointestinal and urological symptoms soon after getting a prostate MRI to workup an elevated PSA level.

On arrival, he was afebrile and hemodynamically stable. Labs were remarkable for an INR of 12.0, a WBC of 18.6, a creatinine of 1.61 (baseline 1.3), and urinalysis showing 3+ blood and 111 red blood cells. An abdomen and pelvis CT without contrast showed marked circumferential wall thickening of a $12.5 \mathrm{~cm}$ length of mid small bowel with evidence of intraluminal bleeding, surrounding free fluid and wall stranding but no significant mural enhancement, concerning for spontaneous intramural hematoma. It also showed a hypo enhancement in the upper left ureteral pole, consistent with a thrombus, left hydroureteronephrosis and thickening of the right renal collecting system, possibly in the setting of hemorrhage. He was given a prothrombin complex concentrate (KCentra) in the emergency department and admitted for further management. He was originally prescribed warfarin to reduce the risk of paradoxical emboli, which had caused a prior stroke with residual minor word finding deficits. His last INR checked four weeks prior to admission was 2.5 , within therapeutic range as it had been at each four-week follow up for the prior six months. Other than elevated PSA level detected at a primary care visit, he had no major medical illnesses, surgeries, or new diagnoses over the past year. He endorsed poor PO intake on the day of his MRI but otherwise had no dietary changes. He had no new medications and his warfarin had been stable at 5 milligrams daily for the past six months. He drank socially, reporting an intake of two beers a month since age 18. He had not smoked tobacco for twenty years. He reported no recreational drug use except for marijuana, which he began using about one month prior. He ingested nightly "marijuana honey," made using cannabis purchased from a local dispensary. His wife estimated he used "an eighth" or 3.5 grams of cannabis weekly. After withholding his warfarin for one day and administering KCentra, the INR improved to 0.9 and remained normal through the remaining part of his hospitalization. His pain, hematuria, and constipation had all resolved before he was discharged. His cardiologist advised that an alternative anticoagulation strategy should be adopted if he continued to use marijuana. He ultimately elected to remain off warfarin and have a minimally invasive closure of his PFO. He continues to consume marijuana honey as of his last recorded outpatient visit. A literature review was conducted on PubMed using the terms "(marijuana) AND warfarin," "(cannabis) AND warfarin," "(marijuana) AND INR," and "(cannabis) AND INR," which yielded nine articles consisting of case reports, systematic reviews, and in vitro studies [1-8]. 
One article was excluded as it was written in Polish. Among those included is a study detailing a March to April 2018 coagulopathy outbreak in the setting of synthetic cannabinoid use tainted with brodifacoum, a vitamin $\mathrm{K}$ antagonist used as rodenticide [1]. A 1997 case report similarly described brodifacoum laced marijuana causing vitamin-K dependent coagulopathy [2]. However, the review also included three case reports that suggest a temporal relationship between natural cannabis use and elevated INR among warfarin users. One 2009 report presented the case of a 56-yearold male on warfarin for eleven years following mechanical heart valve replacement who presented with gastrointestinal bleeding and an INR of 10.41 [3]. He re-presented to the same hospital fifteen days after discharge with epistaxis, easy bruising and an INR of 11.55. During the time between hospitalizations, he endorsed increasing inhaled cannabis use due to worsening depression. He stopped using marijuana for nine months during which time his INR remained below 4.40. A 2019 report described a 35-year-old male who had been stable on warfarin for eight years until his INR rose to 7.2 after a month of using edible and inhaled cannabis [4]. After stopping cannabis and adjusting his warfarin dose, his INR remained below [4].

Several of these studies suggest that cannabis, inhibits cytochrome P450 enzyme CYP2C9, which mediates warfarin metabolism [5-7]. One review that examined the role of cannabis for epilepsy treatment, proposed that CYP29C19 was inhibited by cannabinol (CBD), a non-psychoactive component of cannabis [6]. Additionally, a 2012 in vitro study found that CBD, Delta9tetrahydrocannabinol (THC), and cannabinol (CBN) all potently inhibited CYP2C9 in human liver samples [8]. Notably, these inhibitory effects occurred in a concentration-dependent manner. This case was a unique clinical presentation that members and consultants of the care team had never before encountered. However, as evidenced by a literature review, similar cases have emerged and will likely continue to do so in the trend of increasing cannabis use in the US. We hope to raise clinical awareness of a serious potential interaction between these two commonly used substances, to educate patients prescribed warfarin and to initiate early interventions in the events of acute intoxication. Promotion of public awareness may hopefully prevent morbidity and mortality for many people within the US and beyond. Further studies will be important to elucidate the quantity and type of cannabis, as well as form of ingestion that can trigger this harmful drug interaction to provide future guidelines for practitioners.

\section{References}

1. Ross CH, Singh P, Simon EL (2019) Hemorrhagic soft tissue upper airway obstruction from brodifacoum-contaminated synthetic cannabinoid. J Emerg Med 57(1): 47-50.

2. La Rosa FG, Clarke SH, Lefkowitz JB (1997) Brodifacoum intoxication with marijuana smoking. Arch Pathol Lab Med 121(1): 67-69.

3. Yamreudeewong W, Wong HK, Brausch LM, Pulley KR (2009) Probable interaction between warfarin and marijuana smoking. Ann Pharmacother 43(7): 1347-1353.

4. Hsu A, Painter NA (2019) Probable interaction between warfarin and inhaled and oral administration of cannabis. J Pharm Pract 18: 897190019854958.

5. Damkier P, Lassen D, Christensen MMH, Madesen KG (2019) Interaction between warfarin and cannabis. Basic Clin Pharmacol Toxicol 124(1): 28-31.

6. Gaston TE, Szaflarski JP (2018) Cannabis for the treatment of epilepsy. Curr Neurol Neurosci Rep 18(11): 73.

7. Yamaori S, Koeda K, Kushihara M, Hada Y (2012) Comparison in the in vitro inhibitory effects of major phytocannabinoids and polycyclic aromatic hydrocarbons contained in marijuana smoke on cytochrome P450 2C9 activity. Drug Metab Pharmacokinet 27(3): 294-300.

8. Ge B, Zhang Z, Zuo Z (2014) Updates on the clinical evidenced herbwarfarin interactions. Evid Based Complement Alternat Med: 957362.

For possible submissions Click below: 